\title{
Global Hopf Bifurcation Analysis for an Avian Influenza Virus Propagation Model with Nonlinear Incidence Rate and Delay
}

\author{
Yanhui Zhai, Ying Xiong, Xiaona Ma, and Haiyun Bai \\ School of Science, Tianjin Polytechnic University, Tianjin 300387, China \\ Correspondence should be addressed to Ying Xiong; 542790840@qq.com
}

Received 1 January 2014; Accepted 14 June 2014; Published 14 July 2014

Academic Editor: Zhichun Yang

Copyright ( 2014 Yanhui Zhai et al. This is an open access article distributed under the Creative Commons Attribution License, which permits unrestricted use, distribution, and reproduction in any medium, provided the original work is properly cited.

\begin{abstract}
The paper investigated an avian influenza virus propagation model with nonlinear incidence rate and delay based on SIR epidemic model. We regard delay as bifurcating parameter to study the dynamical behaviors. At first, local asymptotical stability and existence of Hopf bifurcation are studied; Hopf bifurcation occurs when time delay passes through a sequence of critical values. An explicit algorithm for determining the direction of the Hopf bifurcations and stability of the bifurcation periodic solutions is derived by applying the normal form theory and center manifold theorem. What is more, the global existence of periodic solutions is established by using a global Hopf bifurcation result.
\end{abstract}

\section{Introduction}

In March 2013, new avian-origin influenza $A(H 7 N 9)$ virus $(A-O I V)$ broke out in Shanghai and the surrounding provinces of China [1]. During the first week of April, this virus had been detected in six provinces and municipal cities; this virus has caused global concern as a potential pandemic threat [2]. The virus fast took people's life without timely treatment. Therefore, strong measures should be taken to control the spread of H7N9 viruses.

H7N9 is an infectious disease caused by influenza A virus. Moreover, it is essential to study and to dominate the spread of H7N9. Mathematical models become important instruments in the analysis and control of infectious diseases. The present study evaluates the possible application of SIR model for H7N9 spreading.

Let $S(t), I(t)$, and $R(t)$ be the population densities of susceptible, infective, and recovered, respectively. Recruitment of new individuals is into the susceptible class at a constant rate $B$ [3]. Parameters $\mu_{1}, \mu_{2}$, and $\mu_{3}$ are positive constants which represent the death rate of the classes, respectively. $\tau$ is the length of the infectious period; $1 / \gamma$ is the average time spent in class $I$ before recovery [3].

In 1979, Cooke [4] used mass action incidence $\beta S(t) I(t-$ $\tau)$. In 2009, Xu and Ma [5] developed the model with the force of infection given by $\beta S(t)(I(t-\tau) /(1+\alpha I(t-\tau)))$, where $\alpha$ determines the level at which the force of infection saturates and $\beta$ is a contract [5]. Then, the avian influenza virus propagation model based on SIR model has the following form:

$$
\begin{gathered}
\dot{S}(t)=B-\mu_{1} S(t)-\frac{\beta S(t) I(t-\tau)}{1+\alpha I(t-\tau)}, \\
\dot{I}(t)=\frac{\beta S(t) I(t-\tau)}{1+\alpha I(t-\tau)}-\left(\mu_{2}+\gamma\right) I(t), \\
\dot{R}(t)=\gamma I(t)-\mu_{3} R(t) .
\end{gathered}
$$

Since $R$ does not appear in the first two equations, and avoid excessive use of parentheses in some of the latter calculations, the avian influenza virus propagation model is transformed into the following form

$$
\begin{gathered}
\dot{S}(t)=B-\mu_{1} S(t)-\frac{\beta S(t) I(t-\tau)}{1+\alpha I(t-\tau)}, \\
\dot{I}(t)=\frac{\beta S(t) I(t-\tau)}{1+\alpha I(t-\tau)}-\left(\mu_{2}+\gamma\right) I(t), \\
\dot{R}(t)=\gamma I(t)-\mu_{3} R(t),
\end{gathered}
$$


with the following initial condition:

$$
\begin{array}{r}
S(0) \in R^{+}, I(\theta)=\phi(\theta) \quad \text { for } \theta \in[-\tau, 0], \\
\text { where } \phi \in C\left([-\tau, 0], R^{+}\right),
\end{array}
$$

which was presented and studied in [3].

The steady state of the model and the stability of epidemic models have been studied in many papers. Zhang and $\mathrm{Li}$ [6] studied the global stability of an SIR epidemic model with constant infectious periods. $\mathrm{Xu}$ and $\mathrm{Ma}$ [5] showed the global stability of the endemic equilibrium for the case of the reproduction number $R_{0}>1$. McCluskey [3] shown that the endemic equilibrium is globally asymptotically stable whenever it exists. In this paper, we investigated the Hopf bifurcation and the global existence of periodic solutions of model (2), which have not been reported yet.

The organization of this paper is as follows. In Section 2, we will investigate the local asymptotical stability and existence of Hopf bifurcation by analyzing the associated characteristic equation. In Section 3, an explicit algorithm for determining the direction of the Hopf bifurcations and stability of the bifurcation periodic solutions will be derived by applying the normal form theory and center manifold theorem. In Section 4, existence of global periodic solutions will be established by using a global Hopf bifurcation result. In Section 5, a brief discussion is offered to conclude this work.

\section{Local Stability and Hopf Bifurcation}

Some results can be directly obtained from $[3,5]$. The basic reproduction number for the model is $R_{0}=B \beta / \mu_{1}\left(\mu_{2}+\right.$ $\gamma)$. System (2) always has a disease-free equilibrium $E_{1}=$ $\left(B / \mu_{1}, 0\right)$. If $B \beta>\mu_{1}\left(\mu_{2}+\gamma\right)$, system (2) has a unique endemic equilibrium $E^{*}=\left(S^{*}, I^{*}\right)=\left(\left(B \alpha+\mu_{2}+\gamma\right) /\left(\beta+\alpha \mu_{1}\right),(B \beta-\right.$ $\left.\left.\mu_{1}\left(\mu_{2}+\gamma\right)\right) /\left(\mu_{2}+\gamma\right)\left(\beta+\alpha \mu_{1}\right)\right)$ [3]. The characteristic equation of system (2) at the endemic equilibrium $E^{*}$ is

$$
\lambda^{2}+p_{1} \lambda+p_{0}+\left(q_{1} \lambda+q_{0}\right) e^{-\lambda \tau}=0
$$

where $p_{0}=\left(\mu_{2}+\gamma\right)\left(\mu_{1}+\beta I^{*} /\left(1+\alpha I^{*}\right)\right), p_{1}=\mu_{1}+\mu_{2}+$ $\gamma+\beta I^{*} /\left(1+\alpha I^{*}\right), q_{0}=-\beta \mu_{1} S^{*} /\left(1+\alpha I^{*}\right)^{2}$, and $q_{1}=-\beta S^{*} /$ $\left(1+\alpha I^{*}\right)^{2}$. If

$$
R_{0}>1
$$

hold, when $\tau=0$, the endemic equilibrium $E^{*}$ of system (2) is locally stable [5].

If $i \omega(\omega>0)$ is a solution of system (2), separating real and imaginary parts, we obtain the following:

$$
\begin{gathered}
p_{1} \omega=q_{0} \sin \omega \tau-q_{1} \omega \cos \omega \tau, \\
\omega^{2}-p_{0}=q_{0} \cos \omega \tau+q_{1} \omega \sin \omega \tau .
\end{gathered}
$$

Then, we get

$$
\begin{aligned}
& \cos \omega \tau=\frac{\left(q_{0}-p_{1} q_{1}\right) \omega^{2}-p_{0} q_{0}}{q_{0}^{2}+q_{1}^{2} \omega^{2}}, \\
& \sin \omega \tau=\frac{p_{1} q_{0} \omega+\left(\omega^{2}-p_{0}\right) q_{1} \omega}{q_{0}^{2}+q_{1}^{2} \omega^{2}} .
\end{aligned}
$$

It follows that

$$
\omega^{4}+\left(p_{1}^{2}-2 p_{0}-q_{1}^{2}\right) \omega^{2}+p_{0}^{2}-q_{0}^{2}=0 .
$$

Letting $z=\omega^{2}$, we get

$$
z^{2}+\left(p_{1}^{2}-2 p_{0}-q_{1}^{2}\right) z+p_{0}^{2}-q_{0}^{2}=0
$$

It is easy to show that

$$
\begin{aligned}
& p_{1}^{2}-2 p_{0}-q_{1}^{2}=\left(\mu_{1}+\frac{\beta I^{*}}{1+\alpha I^{*}}\right)^{2} \\
& \quad+\left(\mu_{2}+\gamma\right)^{2}-\frac{\left(\mu_{2}+\gamma\right)^{2}}{\left(1+\alpha I^{*}\right)^{2}}>0, \\
& p_{0}^{2}-q_{0}^{2} \\
& =\left(\mu_{2}+\gamma\right)\left[\left(\mu_{2}+\gamma\right)\left(\mu_{1}+\frac{\beta I^{*}}{1+\alpha I^{*}}\right)+\frac{\beta \mu_{1} S^{*}}{\left(1+\alpha I^{*}\right)^{2}}\right] \\
& \quad \times\left(\mu_{1}-\frac{\mu_{1}}{1+\alpha I^{*}}+\frac{\beta I^{*}}{1+\alpha I^{*}}\right) .
\end{aligned}
$$

The case of

$$
\beta \geq \mu_{1} \alpha
$$

has been discussed in [5]. We obtain global asymptotic stability of the endemic equilibrium when $R_{0}>1$. If

$$
\beta<\mu_{1} \alpha
$$

hold, that is, $\left(\beta-\mu_{1} \alpha\right) I^{*} /\left(1+\alpha I^{*}\right)<0$, we have $p_{0}^{2}-q_{0}^{2}<0$. Following the theorem given by Ruan [7], there exists critical value

$$
\tau_{k}^{(j)}=\frac{1}{\omega_{k}} \arccos \frac{\left(\omega_{k}^{2}-p_{0}\right) q_{0}-p_{1} q_{1} \omega_{k}^{2}}{q_{0}^{2}+q_{1}^{2} \omega_{k}^{2}}+\frac{2 j \pi}{\omega_{k}},
$$

with

$\omega_{j}$

$$
=\left[\frac{2 p_{0}+p_{1}^{2}-q_{1}^{2}+\sqrt{\left(2 p_{0}+p_{1}^{2}-q_{1}^{2}\right)^{2}-4\left(p_{0}^{2}-q_{0}^{2}\right)}}{2}\right]^{1 / 2},
$$

where $k=1,2, \ldots, j=0,1,2, \ldots$. If $\left(P_{1}\right)$ and $\left(H_{2}\right)$ are satisfied, (6) has a pair of purely imaginary roots $\pm \omega_{0} i$ when 
$\tau=\tau_{0}$. Additionally, all roots of (6) have negative real parts when $\tau \in\left[0, \tau_{0}\right]$ and when $\tau>\tau_{0}$ (5) has at least a pair of roots with positive real part. In order to give the main results, it is necessary to prove the transversality condition $\operatorname{Re}(d \lambda / d \tau)^{-1}>0$ holds. Denote $\lambda=\alpha(\tau)+i \omega(\tau)$ as the root of (5) with $\alpha(\tau)=0, \omega(\tau)=\omega_{0}$. Differentiating (5) with respect to $\tau$ yields

$$
\left[2 \lambda+p_{1}+\left(q_{1}-\tau\left(q_{1} \lambda+q_{0}\right) e^{-\lambda \tau}\right)\right] \frac{d \lambda}{d \tau}=\lambda\left(q_{1} \lambda+q_{0}\right) e^{-\lambda \tau} .
$$

For the sake of simplicity denoting $\omega_{0}$ and $\tau_{0}$ by $\omega, \tau$, respectively,

$$
\frac{d \lambda}{d \tau}=\frac{\left(q_{1} \lambda+q_{0}\right) \lambda e^{-\lambda \tau}}{2 \lambda+p_{1}+q_{1} e^{-\lambda \tau}-\left(\left(q_{1} \lambda+q_{0}\right) \tau e^{-\lambda \tau}\right)}
$$

in the following:

$$
\begin{aligned}
& \operatorname{Re}\left(\frac{d \lambda}{d \tau}\right)^{-1} \\
& =\frac{2 \lambda+p_{1}+q_{1} e^{-\lambda \tau}}{\left(q_{1} \lambda+q_{0}\right) \lambda e^{-\lambda \tau}} \\
& =\left(\left(p_{1} \cos \omega \tau-2 \omega \sin \omega \tau+q_{1}\right)\right. \\
& \left.+i\left(2 \omega \cos \omega \tau+p_{1} \sin \omega \tau\right)\right) \\
& \times\left(-q_{1} \omega^{2}+i q_{0} \omega\right)^{-1} \\
& =\left(-p_{1} q_{1} \omega^{2} \cos \omega \tau+2 q_{1} \omega^{3} \sin \omega \tau\right. \\
& \left.-q_{1}^{2} \omega^{2}+2 q_{0} \omega^{2} \cos \omega \tau+p_{1} q_{0} \omega \sin \omega \tau\right) \\
& \times\left(q_{0} \omega^{2}+\omega^{4}\right)^{-1} \\
& =\left(\left(-p_{1} q_{1} \omega^{2}+2 q_{0} \omega^{2}\right)\left(q_{0}-p_{1} q_{1}\right) \omega^{2}-p_{0} q_{0}\right. \\
& +\left(2 q_{1} \omega^{3}+p_{1} q_{0} \omega\right) \\
& \left.\times\left[p_{1} q_{0} \omega+\left(\omega^{2}-p_{0}\right) q_{1} \omega\right]-q_{0}^{2} q_{1}^{2} \omega^{2}-q_{1}^{4} \omega^{2}\right) \\
& \times\left(\left(q_{0}^{2}+q_{1}^{2} \omega^{2}\right)\left(q_{0} \omega^{2}+\omega^{4}\right)\right)^{-1} \\
& =\left(2 q_{1}^{2} \omega^{6}+\left[2 q_{0}^{2}+\left(p_{1}^{2}-2 p_{0}-q_{1}^{2}\right) q_{1}^{2}\right] \omega^{4}\right. \\
& \left.+\left(p_{1}^{2}+2 p_{0}-q_{1}^{2}\right) q_{0}^{2} \omega^{2}\right) \\
& \times\left(\left(q_{0}^{2}+q_{1}^{2} \omega^{2}\right)\left(q_{0} \omega^{2}+\omega^{4}\right)\right)^{-1} .
\end{aligned}
$$

From (10), we know $p_{1}^{2}-2 p_{0}-q_{1}^{2}>0$; then, $\operatorname{Re}(d \lambda / d \tau)^{-1}>0$ hold. Under this condition, we have the following theorem.

Theorem 1. (i) If $\left(P_{1}\right)$ and $\left(H_{1}\right)$ holds, the equilibrium $\left(S^{*}, I^{*}\right)$ of system $(2)$ is asymptotically stable for any $\tau>0$.

(ii) If $\left(P_{1}\right)$ and $\left(H_{2}\right)$ holds, $\left(S^{*}, I^{*}\right)$ is asymptotically stable for $\tau \in\left[0, \tau_{0}\right)$ and unstable for $\tau \in\left(\tau_{0},+\infty\right)$. System (2) exhibits the Hopf bifurcation at the equilibrium $\left(S^{*}, I^{*}\right)$ for $\tau=\tau_{j}, j=0,1,2, \ldots$

\section{Direction and Stability of the Bifurcating Periodic Solutions}

In Section 2, we obtain the conditions under which a family of periodic solutions bifurcate from the steady state at the critical value of $\tau$. In this section, we investigate the direction of the Hopf bifurcation and the stability of the bifurcating periodic solution at critical values $\tau_{0}$, using techniques of the normal form theory and center manifold theorem.

Let $u_{1}=S(t)-S^{*}$ and let $u_{2}=I(t)-I^{*}$. The Taylor expansion of system (2) at $E^{*}$ is

$$
\begin{aligned}
\dot{u}_{1}(t)= & a_{1} u_{1}(t)-a_{2} u_{2}(t-\tau) \\
& +a_{6} u_{2}(t)^{2}(t-\tau)+a_{7} u_{1}(t) u_{2}(t-\tau), \\
\dot{u}_{2}(t)= & a_{3} u_{1}(t)+a_{4} u_{2}(t)+a_{5} u_{2}(t-\tau) \\
& -a_{7} u_{1}(t) u_{2}(t-\tau)-a_{6} u_{2}^{2}(t-\tau),
\end{aligned}
$$

where $a_{1}=-\mu_{1}+\beta I^{*} /\left(1+\alpha I^{*}\right), a_{2}=-\beta S^{2} /\left(1+\alpha I^{*}\right)$, $a_{3}=\beta I^{*} /\left(1+\alpha I^{*}\right), a_{4}=-\mu_{2}-\gamma, a_{5}-\beta S^{2} /\left(1+\alpha I^{*}\right)$, $a_{6}=-\alpha \beta S^{*} /\left(1+\alpha I^{*}\right)^{3}, a_{7}=\beta /\left(1+\alpha I^{*}\right)^{2}, \tau=\tau_{0}+\mu$, and $u_{t}=u(t+\theta) \in C_{1}$ for $\theta \in[-1,0]$. System (2) is transformed into FDE as

$$
\dot{u}(t)=L_{\mu}+F\left(u_{t}, \mu\right)
$$

with

$$
\begin{gathered}
L_{\mu}(\phi)=\left(\tau_{0}+\mu\right)\left[B_{1} \phi(0)+B_{2} \phi(-1)\right], \\
F(\phi, \mu)=\left(\tau_{0}+\mu\right)\left(\begin{array}{c}
a_{6} \phi_{2}^{2}(-1)+a_{7} \phi_{1}(0) \phi_{2}(-1) \\
-a_{6} \phi_{1}(0) \phi_{2}(-1)-a_{7} \phi_{2}^{2}(-1)
\end{array}\right),
\end{gathered}
$$

where

$$
B_{1}=\left(\begin{array}{cc}
a_{1} & 0 \\
a_{3} & a_{4}
\end{array}\right), \quad B_{2}=\left(\begin{array}{ll}
0 & a_{2} \\
0 & a_{5}
\end{array}\right) .
$$

By Riesz representation theorem, there exists a function $\eta(\theta, \mu)$ of bounded variation, for $\theta \in[-1,0]$, such that

$$
L_{\mu} \phi=\int_{-1}^{0} d \eta(\theta, \mu) \phi(\theta) \quad \text { for } \phi \in C .
$$

In fact, we can choose

$$
\eta(\theta, \mu)=\left(\tau_{0}+\mu\right)\left[B_{1} \delta(\theta)+B_{2} \delta(\theta+1)\right],
$$

where $\delta(\theta)$ is a delta function.

For $\phi \in C^{\prime}[-1,0]$, the operators $A$ and $R$ are defined as follows:

$$
\begin{gathered}
A(\mu) \phi(\theta)= \begin{cases}\frac{d \phi(\theta)}{d \theta}, & \theta \in[-1,0), \\
\int_{-1}^{0} d(\eta(t, \mu) \phi(t)), & \theta=0,\end{cases} \\
R(\mu) \phi(\theta)= \begin{cases}0, & \theta \in[-1,0), \\
f(\mu, \theta), & \theta=0 .\end{cases}
\end{gathered}
$$


The adjoint operator $A^{*}(0)$ corresponding to $A(0)$ is defined as follows:

$$
A^{*} \psi(s)= \begin{cases}-\frac{d \psi(s)}{d s}, & s \in(0,1], \\ \int_{-1}^{0} d\left(\eta^{T}(t, 0) \psi(-t)\right), & s=0\end{cases}
$$

and an adjoint bilinear is as follows:

$$
\langle\psi, \phi\rangle=\bar{\psi}(0) \phi(0)-\int_{-1}^{0} \int_{0}^{\theta} \bar{\psi}(\xi-\theta) d \eta(\theta) \phi(\xi) d \xi
$$

where $\eta(\theta)=\eta(\theta, 0)$.

From the preceding discussion, we know that $q(\theta)$ and $q^{*}(\theta)$ be the eigenvectors of $A$ and $A^{*}$ corresponding to $i \tau_{0} \omega_{0}$ and $-i \tau_{0} \omega_{0}$, respectively. Next, we calculate $q(\theta)$ and $q^{*}(s)$ to determine the normal form of operator $A$.

Proposition 2. Let $q(\theta)$ and $q^{*}(s)$ be eigenvectors of $A$ and $A^{*}$ corresponding to $i \tau_{0} \omega_{0}$ and $-i \tau_{0} \omega_{0}$, respectively, satisfying $\left\langle q^{*}, q\right\rangle=1$ and $\left\langle q^{*}, \bar{q}\right\rangle=0$.

Then,

$$
\begin{gathered}
q(\theta)=(1, \alpha)^{T} e^{i \omega_{0} \tau_{0} \theta}=\left(1, \frac{\omega_{0} i-a_{1}}{a_{2} e^{-i \omega_{0} \tau_{0}}}\right)^{T} e^{i \omega_{0} \tau_{0} \theta}, \\
q^{*}(s)=D\left(1, \beta^{*}\right) e^{-i \omega_{0} \tau_{0} s}=\left(1, \frac{-\omega_{0} i-a_{1}}{a_{3}}\right)^{T} e^{-i \omega_{0} \tau_{0} s},
\end{gathered}
$$

where

$$
D=\frac{1}{1+\alpha \bar{\beta}^{*}-\tau_{0} \alpha\left(a_{2}+\bar{\beta}^{*} a_{5}\right) e^{-i \omega_{0} \tau_{0}}} .
$$

Proof. Without loss of generality, we just consider the eigenvector $q(\theta)$. By the definition of $A$ and $q(\theta)$ with $\theta \in[-1,0)$, we get $q(\theta)=(1, \alpha)^{T} e^{i \omega_{0} \tau_{0}}$ (here, $\alpha$ is a parameter). In what follows, notice that $q(0)=(1, \alpha)^{T}$ and $A q(0)=$ $\int_{-1}^{0} d(\eta(t, \mu) \phi(t))=i \omega_{0} \tau_{0} q(0)$; we have $\alpha=\left(\omega_{0} i-\right.$ $\left.a_{1}\right) / a_{2} e^{-i \omega_{0} \tau_{0}}$. Using a proof procedure similar to that in [8], by direct computation, we get $q(\theta)$ and $q^{*}(s)$. Bring $q(\theta)$ and $q^{*}(s)$ into $\left\langle q^{*}, q\right\rangle=1$; it is not hard to obtain the parameter $\bar{D}$. The detailed procedure of proof refers to [9]. The proof is completed.

Then, we construct the coordinates of the center manifold $C_{0}$ at $\mu=0$. Let

$$
z(t)=\left\langle q^{*}, u_{t}\right\rangle, \quad W(t, \theta)=u_{t}(\theta)-2 \operatorname{Re}\{z(t) q(\theta)\} .
$$

On the center manifold $C_{0}$, we have

$$
W(t, \theta)=W(z(t), \overline{z(t)}, \theta)
$$

where

$$
W(z, \bar{z}, \theta)=W_{20}(\theta) \frac{z^{2}}{2}+W_{11}(\theta) z \bar{z}+W_{02} \frac{\bar{z}^{2}}{2}+W_{30} \frac{z^{3}}{6}, \ldots ;
$$

and $z$ and $\bar{z}$ are local coordinates for the center manifold $C_{0}$ in the direction of $q$ and $q^{*}$, respectively. Since $\mu=0$, we have

$$
\begin{aligned}
z^{\prime}(t) & =i \tau_{0} \omega_{0} z(t)+\left\langle q^{*}(\theta), f(W+2 \operatorname{Re}\{z(t) q(\theta)\})\right\rangle \\
& =i \tau_{0} \omega_{0} z(t)+\overline{q^{*}(0)} f(W(z, \bar{z}, 0)+2 \operatorname{Re}\{z(t) q(0)\}) \\
& \triangleq i \tau_{0} \omega_{0} z(t)+\overline{q^{*}(0)} f_{0}(z, \bar{z}),
\end{aligned}
$$

where

$$
f_{0}(z, \bar{z})=f_{z^{2}} \frac{z^{2}}{2}+f_{\bar{z}^{2}} \frac{\bar{z}^{2}}{2}+f_{z \bar{z}} z \bar{z}+\cdots
$$

We rewrite this as

$$
z^{\prime}(t)=i \tau_{0} \omega_{0} z+g(z, \bar{z})
$$

with

$$
\begin{gathered}
g(z, \bar{z})=\overline{q^{*}}(0) f_{0}(z, \bar{z}) \\
=g_{20} \frac{z^{2}}{2}+g_{11} z \bar{z}+g_{02} \frac{\bar{z}^{2}}{2}+g_{21} \frac{z^{2} \bar{z}}{2}+\cdots, \\
g(z, \bar{z})=D \tau_{0}\left(1, \overline{\beta^{*}}\right)\left(\begin{array}{c}
a_{6} \phi_{2}^{2}(-1)+a_{7} \phi_{1}(0) \phi_{2}(-1) \\
-a_{6} \phi_{1}(0) \phi_{2}(-1)-a_{7} \phi_{2}^{2}(-1)
\end{array}\right),
\end{gathered}
$$

where

$$
\begin{aligned}
\phi_{1}(0)= & z+\bar{z}+W_{20}^{(1)}(0) \frac{z^{2}}{2}+W_{11}^{(1)}(0) z \bar{z} \\
& +W_{02}^{(1)}(0) \frac{\bar{z}}{2}, \\
\phi_{2}(-1)= & z \alpha e^{-i \omega_{0} \tau_{0}}+\bar{z} \bar{\alpha} e^{i \omega_{0} \tau_{0}}+W_{20}^{(2)}(-1) \frac{z^{2}}{2} \\
& +W_{11}^{(2)}(-1) z \bar{z}+W_{02}^{(2)}(-1) \frac{\bar{z}}{2} .
\end{aligned}
$$

Comparing the coefficients of the above equation with (22), we obtain

$$
\begin{aligned}
& g_{20} \\
& =2 D \tau_{0}\left[\left(a_{6}-\overline{\beta^{*}} a_{7}\right) \alpha^{2} e^{-2 i \omega_{0} \tau_{0}}+\left(a_{7}-\overline{\beta^{*}} a_{6}\right) \alpha e^{-i \omega_{0} \tau_{0}}\right],
\end{aligned}
$$

$g_{11}$

$$
\begin{aligned}
=D \tau_{0}[2 & \left(a_{6}-\overline{\beta^{*}} a_{7}\right) \alpha \bar{\alpha}+\left(a_{7}-\overline{\beta^{*}} a_{6}\right) \\
& \left.\times\left(\bar{\alpha} e^{i \omega_{0} \tau_{0}}+\alpha e^{-i \omega_{0} \tau_{0}}\right)\right],
\end{aligned}
$$

$g_{02}$

$=2 D \tau_{0}\left[\left(a_{6}-\overline{\beta^{*}} a_{7}\right) \overline{\alpha^{2}} e^{2 i \omega_{0} \tau_{0}}\right.$

$$
\left.+\left(a_{7}-\overline{\beta^{*}} a_{6}\right) \bar{\alpha} e^{-i \omega_{0} \tau_{0}}\right],
$$




$$
\begin{aligned}
& g_{21}=2 D \tau_{0}\left[\left(a_{6}-\overline{\beta^{*}} a_{7}\right)\right. \\
& \times\left[\bar{\alpha} e^{i \omega_{0} \tau_{0}} W_{20}^{(2)}(-1)+2 \alpha e^{-i \omega_{0} \tau_{0}} W_{11}^{(2)}(-1)\right] \\
& +\left(a_{7}-\overline{\beta^{*}} a_{6}\right) \\
& \times\left[\frac{1}{2} \bar{\alpha} e^{i \omega_{0} \tau_{0}} W_{20}^{(1)}(0)+\alpha e^{-i \omega_{0} \tau_{0}} W_{11}^{(1)}(0)\right. \\
& \left.+\frac{1}{2} W_{20}^{(2)}(-1)+W_{11}^{(2)}(-1)\right], \\
& \dot{W}=\dot{u}_{t}-\dot{z} q-\dot{\bar{z}} \bar{q} \\
& = \begin{cases}A W-2 \operatorname{Re} \overline{q^{*}}(0) f_{0} q(\theta), & \theta \in[-1,0], \\
A W-2 \operatorname{Re} \overline{q^{*}}(0) f_{0} q(\theta)+f_{0}, & \theta=0 .\end{cases} \\
& \triangleq A W+H(z, \bar{z}, \theta) \text {, }
\end{aligned}
$$

where

$$
H(z, \bar{z}, \theta)=H_{20}(\theta) \frac{z^{2}}{2}+H_{11}(\theta) z \bar{z}+H_{02}(\theta) \frac{\bar{z}^{2}}{2}+\cdots .
$$

Expanding the above series and comparing the coefficients, we get

$$
\begin{gathered}
\left(A-2 i \omega_{0} \tau_{0} I\right) W_{20}(\theta)=-H_{20}(\theta), \\
A W_{11}(\theta)=-H_{11}(\theta), \\
\left(A+2 i \omega_{0} \tau_{0} I\right) W_{02}(\theta)=-H_{02}(\theta) .
\end{gathered}
$$

Comparing the coefficients with (38), we obtain

$$
\begin{aligned}
& H_{20}(\theta)=-g_{20} q(\theta)-\bar{g}_{02} \bar{q}(\theta), \\
& H_{11}(\theta)=-g_{11} q(\theta)-\bar{g}_{11} \bar{q}(\theta) .
\end{aligned}
$$
have

It follows from (39), (40), and the definition of $A$ that we

$$
\begin{gathered}
\dot{W}_{20}(\theta)=2 i \tau_{0} \omega_{0} W_{20}(\theta)+g_{20} q(\theta)+\bar{g}_{20} \bar{q}(\theta), \\
\dot{W}_{11}(\theta)=g_{11} q(\theta)+\bar{g}_{11} \bar{q}(\theta) .
\end{gathered}
$$

So,

$$
\begin{gathered}
W_{20}(\theta)=\frac{i g_{20}}{\tau_{0} \omega_{0}} q(0) e^{i \tau_{0} \omega_{0} \theta} \\
-\frac{\bar{g}_{02}}{3 i \tau_{0} \omega_{0}} \bar{q}(0) e^{-i \tau_{0} \omega_{0} \theta}+E_{1} e^{2 i \tau_{0} \omega_{0} \theta}, \\
W_{11}(\theta)=-\frac{i g_{11}}{\tau_{0} \omega_{0}} q(0) e^{i \tau_{0} \omega_{0} \theta}+\frac{i \bar{g}_{11}}{\tau_{0} \omega_{0}} \bar{q}(0) e^{-i \tau_{0} \omega_{0} \theta}+E_{2},
\end{gathered}
$$

Let $C\left(E^{*}, \tau_{j}, 2 \pi / \omega_{0}\right)$ denote the connected component of $C\left(E^{*}, \tau_{j}, 2 \pi / \omega_{0}\right)$ in $\Sigma$ and $\operatorname{Proj}_{\tau}\left(E^{*}, \tau_{j}, 2 \pi / \omega_{0}\right)$ its projection on $\tau$ component. From theorem (5), we know that 
$C\left(E^{*}, \tau_{j}, 2 \pi / \omega_{0}\right)$ is nonempty. $\omega_{0}$ and $\tau_{j}$ are defined in (10) and (11).

Lemma 4. All periodic solutions of system (2) are uniformly bounded.

Proof. Let $(S(t), I(t))$ be a nonconstant periodic solution of system (2), and let $S\left(t_{1}\right), S\left(t_{2}\right)$ and $I\left(t_{3}\right), I\left(t_{4}\right)$ be the maximum and minimum of $S(t)$ and $I(t)$, respectively. Using a proof procedure similar to that in [8], we can obtain

$$
0<S(t)<\frac{B}{\mu_{1}}, \quad 0<I(t)<\frac{B}{\mu_{2}+\gamma} .
$$

It is shown that all periodic solutions of system (2) are uniformly bounded. This completes the proof.

Lemma 5. System (2) has no nonconstant periodic solution of period $\tau$.

Proof. For a contradiction, if system (5) has a $\tau$-periodic solution, say $(S(t), I(t))$, then it satisfies the ODES as follows:

$$
\begin{gathered}
\dot{S}(t)=B-\mu_{1} S(t)-\frac{\beta S(t) I(t)}{1+\alpha I(t)}=P(S, I), \\
\dot{I}(t)=\frac{\beta S(t) I(t)}{1+\alpha I(t)}-\left(\mu_{2}+\gamma\right) I(t)=Q(S, I) .
\end{gathered}
$$

We can get

$$
\frac{\partial P}{\partial S}+\frac{\partial Q}{\partial I}=-\mu_{1}-\frac{\beta I(t)}{1+\alpha I(t)}-\frac{1}{(1+\alpha I(t))^{2}}-\left(\mu_{2}+\gamma\right)<0
$$

By Bendixson's criterion, we know that system (2) has no nonconstant periodic solutions, which prove the lemma.

Theorem 6. Suppose that the condition $\left(H_{1}\right)$ and $\left(P_{1}\right)$ is satisfied. Then, for each $\tau>\tau_{j}, j=0,1,2, \ldots$, system (2) has at least $j-1$ periodic solutions.

Proof. The characteristic matrix of system (2) at the equilib$\operatorname{rium} \bar{z}=\left[\bar{z}^{(1)}, \bar{z}^{(2)}\right] \in R^{2}$ is in the following form:

$$
\Delta(\bar{z}, \tau, l)(\lambda)=\lambda I-D_{\phi} F(\bar{z}, \bar{\tau}, \bar{l})\left(e^{\lambda} I d\right)
$$

that is,

$$
\begin{aligned}
& \Delta(\bar{z}, \tau, l) \\
& =\left(\begin{array}{cc}
\lambda+\mu_{1}+\frac{\beta \bar{z}^{(2)} e^{-\lambda \tau}}{1+\alpha \bar{z}^{(2)} e^{-\lambda \tau}} & \frac{\beta \bar{z}^{(1)}}{\left(1+\alpha \bar{z}^{(2)} e^{-\lambda \tau}\right)^{2}} \\
-\frac{\beta \bar{z}^{(2)} e^{-\lambda \tau}}{1+\alpha \bar{z}^{(2)} e^{-\lambda \tau}} & \lambda+\frac{\beta \bar{z}^{(1)}}{\left(1+\alpha \bar{z}^{(2)} e^{-\lambda \tau}\right)^{2}}
\end{array}\right) .
\end{aligned}
$$

Using a proof procedure similar to that in [9], it is easy to obtain that $\left(E^{*}, \tau_{j}, 2 \pi / \omega_{0}\right), j=0,1,2, \ldots$, is an isolated center.
From the proof procedure of Lemmas 4 and 5, it is easy to know that there exist $\varepsilon>0, \delta>0$, smooth curve $\lambda:\left(\tau_{j}-\right.$ $\left.\delta, \tau_{j}+\delta\right) \rightarrow C$ such that

$$
\Delta(\lambda(\tau))=\Delta_{(v, \tau, T)}(\lambda(\tau))=0, \quad\left|\lambda(\tau)-i \omega_{0}\right|<\varepsilon,
$$

for all $\tau \in\left[\tau_{j}-\delta, \tau_{j}+\delta\right]$, and

$$
\lambda\left(\tau_{j}\right)=i \omega_{0},\left.\quad \frac{d \operatorname{Re}(\lambda(\tau))}{d \tau}\right|_{\tau=\tau_{j}}>0 .
$$

Define $l_{j}=2 \pi / \omega_{0}$, and let $\Omega_{\varepsilon}=\{(0, l): 0<u<\varepsilon, \mid l-$ $\left.l_{j} \mid<\varepsilon\right\}$. Obviously, if $\left|\tau-\tau_{j}\right| \leq \delta$ and $(u, l) \in \partial \Omega_{\varepsilon}$ such that $\Delta_{\left(E^{*}, \tau, l\right)}(u+2 \pi i / l)=0$, if and only if $\tau=\tau_{j}, u=0, l=l_{j}$, set

$$
H^{ \pm}\left(E^{*}, \tau_{j}, \frac{2 \pi}{\omega_{0}}\right)(u, l)=\Delta_{\left(E^{*}, \tau_{j} \pm \delta, l\right)}\left(u+\frac{2 \pi i}{l}\right) .
$$

We obtain the crossing number as follows:

$$
\begin{aligned}
\gamma_{1}\left(E^{*}, \tau_{j}, \frac{2 \pi}{\omega_{0}}\right)= & \operatorname{deg}_{B}\left(H^{-}\left(E^{*}, \tau_{j}, \frac{2 \pi}{\omega_{0}}\right), \Omega_{\varepsilon}\right) \\
& -\operatorname{deg}_{B}\left(H^{+}\left(E^{*}, \tau_{j}, \frac{2 \pi}{\omega_{0}}\right), \Omega_{\varepsilon}\right)=-1
\end{aligned}
$$

We conclude that

$$
\sum_{\left(E^{*}, \tau, l\right) \in C\left(E^{*}, \tau_{j}, 2 \pi / \omega_{0}\right)} \gamma_{1}\left(E^{*}, \tau, l\right)<0
$$

Since the first crossing number of each center is always -1 , by $\left[10\right.$, Theorem 3.3], we conclude that $C\left(E^{*}, \tau_{j}, 2 \pi / \omega_{0}\right)$ is unbounded. By the definition of $\tau_{j}$ given in (10), we know that, for $j \geq 1,\left(\tau_{j} /(j+1)\right)<2 \pi / \omega_{0}<\tau_{j}$ automatically hold.

Again, the population densities of susceptible and infective are ultimately uniformly bounded, implying that the projection of $C\left(E^{*}, \tau_{j}, 2 \pi / \omega_{0}\right)$ onto the $\tau$-space is bounded. Meanwhile, system (2) with $\tau=0$ has no nonconstant periodic solutions; if there exits $\tau^{*}>0$ such that the projection of $C\left(E^{*}, \tau_{j}, 2 \pi / \omega_{0}\right)$ onto the $\tau$-space ins $\left(0, \tau_{0}\right)$ with $\tau^{*}>\tau_{j}$, then, the projection of $C\left(E^{*}, \tau_{j}, 2 \pi / \omega_{0}\right)$ onto the $\tau$-space is bounded. Since $\left(2 \pi / \omega_{0}\right)<\tau_{j}$ and from Lemma 5, we can obtain $0<l<\tau^{*}$ for $(E, \tau, T) \in$ $C\left(E^{*}, \tau_{j}, 2 \pi / \omega_{0}\right)$ with $l<\tau^{*}$; that is to say, $C\left(E^{*}, \tau_{j}, 2 \pi / \omega_{0}\right)$ onto $l$-space is also bounded. Because $C\left(E^{*}, \tau_{j}, 2 \pi / \omega_{0}\right)$ is unbounded, $\operatorname{Proj}_{\tau}\left(E, \tau_{j}, 2 \pi / \omega_{0}\right)$ must be unbounded. Consequently, $\operatorname{Proj}_{\tau}\left(E, \tau_{j}, 2 \pi / \omega_{0}\right)$ include $\left[\tau_{j}, \infty\right)$ for $j \geq 1$. That is to say, for each $\tau>\tau_{j}(j \geq 1)$, system (2) at least has $j-1$ nonconstant period solutions. The proof is complete.

\section{Conclusion}

In this paper we have analytically studied an avian influenza virus propagation model with nonlinear incidence rate and time delay depending on SIR epidemic model. Some previous efforts in epidemic models have been mainly concerned with the global stability and asymptotical stability. However, it 
is a new idea to study the bifurcation periodic solutions and global existence of periodic solutions. The theoretical analysis for the avian influenza virus propagation models is given. Then, Hopf bifurcation occurs when time delay passes through a sequence of critical values. Furthermore, bifurcations and stability of the bifurcation periodic solutions are derived. Finally, global existence of periodic solutions is established.

\section{Conflict of Interests}

The authors declare that they have no financial and personal relationships with other people or organizations that can inappropriately influence their work; there is no professional or other personal interest of any nature or kind in any product, service, and/or company that could be construed as influencing the position presented in, or the review of, in this paper.

\section{References}

[1] J. Li, X. Yu, X. Pu et al., "Environmental connections of novel avian-origin H7N9 influenza virus infection and virus adaptation to the human," Science China Life Sciences, vol. 56, no. 6, pp. 485-492, 2013.

[2] J. He, L. Ning, and Y. Tong, "Origins and evolutionary genomics of the novel 2013 avian-origin H7N9 influenza A virus in China: early findings," http://arxiv.org/abs/1304.1985.

[3] C. C. McCluskey, "Global stability for an SIR epidemic model with delay and nonlinear incidence," Nonlinear Analysis: Real World Applications, vol. 11, no. 4, pp. 3106-3109, 2010.

[4] K. L. Cooke, "Stability analysis for a vector disease model," The Rocky Mountain Journal of Mathematics, vol. 9, no. 1, pp. 31-42, 1979.

[5] R. Xu and Z. Ma, "Global stability of a SIR epidemic model with nonlinear incidence rate and time delay," Nonlinear Analysis: Real World Applications, vol. 10, no. 5, pp. 3175-3189, 2009.

[6] F. Zhang and Z. Li, "Global stability of an SIR epidemic model with constant infectious period," Applied Mathematics and Computation, vol. 199, no. 1, pp. 285-291, 2008.

[7] S. Ruan, "Absolute stability, conditional stability and bifurcation in Kolmogorov-type predator-prey systems with discrete delays," Quarterly of Applied Mathematics, vol. 59, no. 1, pp. 159173, 2001.

[8] J. Wei and C. Yu, "Hopf bifurcation analysis in a model of oscillatory gene expression with delay," Proceedings of the Royal Society of Edinburgh A. Mathematics, vol. 139, no. 4, pp. 879-895, 2009.

[9] X. Meng, H. Huo, and H. Xiang, "Hopf bifurcation in a threespecies system with delays," Journal of Applied Mathematics and Computing, vol. 35, no. 1-2, pp. 635-661, 2011.

[10] J. Wu, "Symmetric functional-differential equations and neural networks with memory," Transactions of the American Mathematical Society, vol. 350, no. 12, pp. 4799-4838, 1998. 


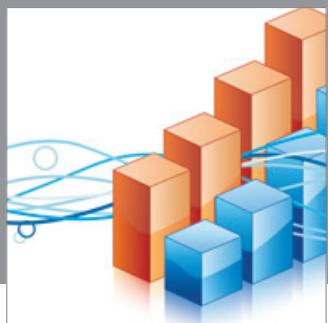

Advances in

Operations Research

mansans

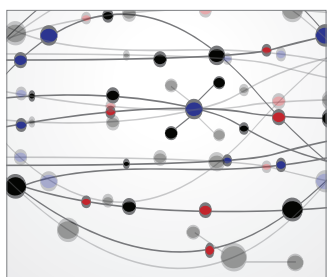

The Scientific World Journal
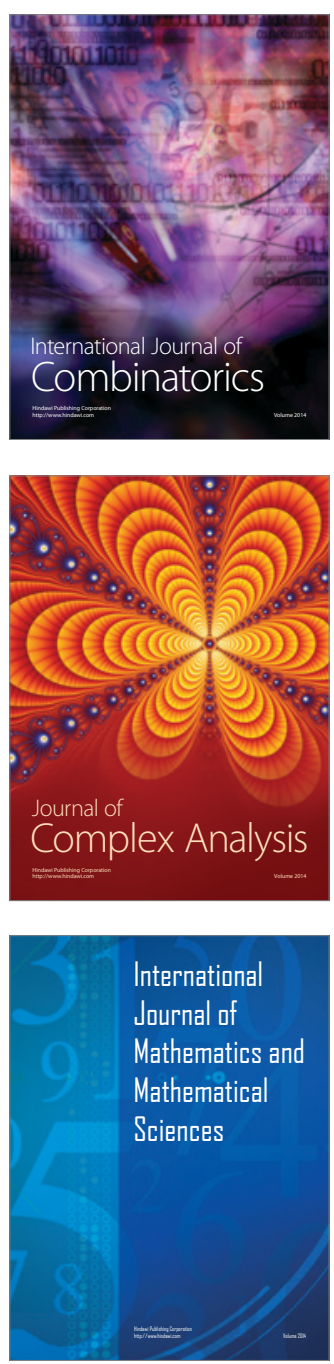
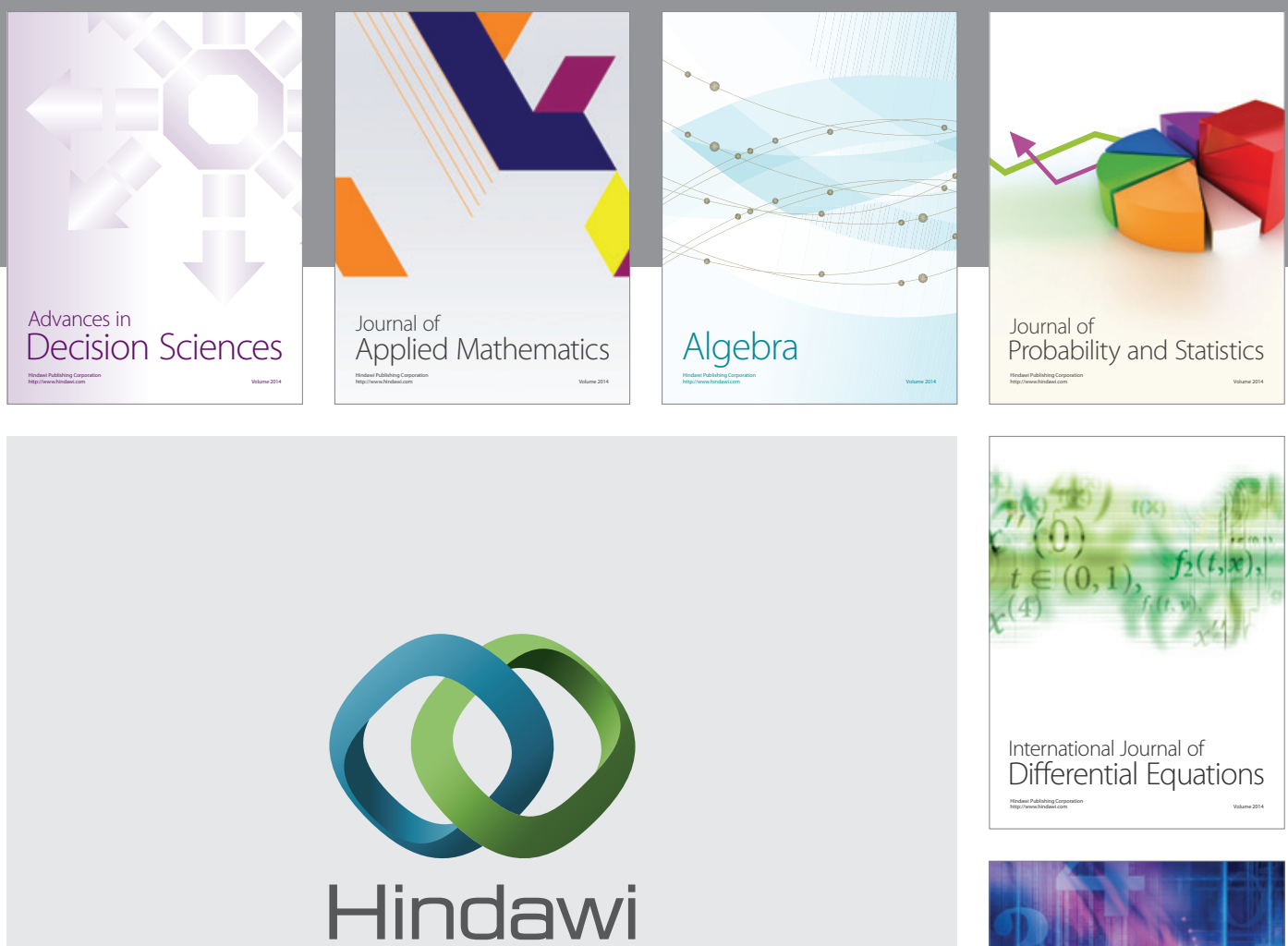

Submit your manuscripts at http://www.hindawi.com
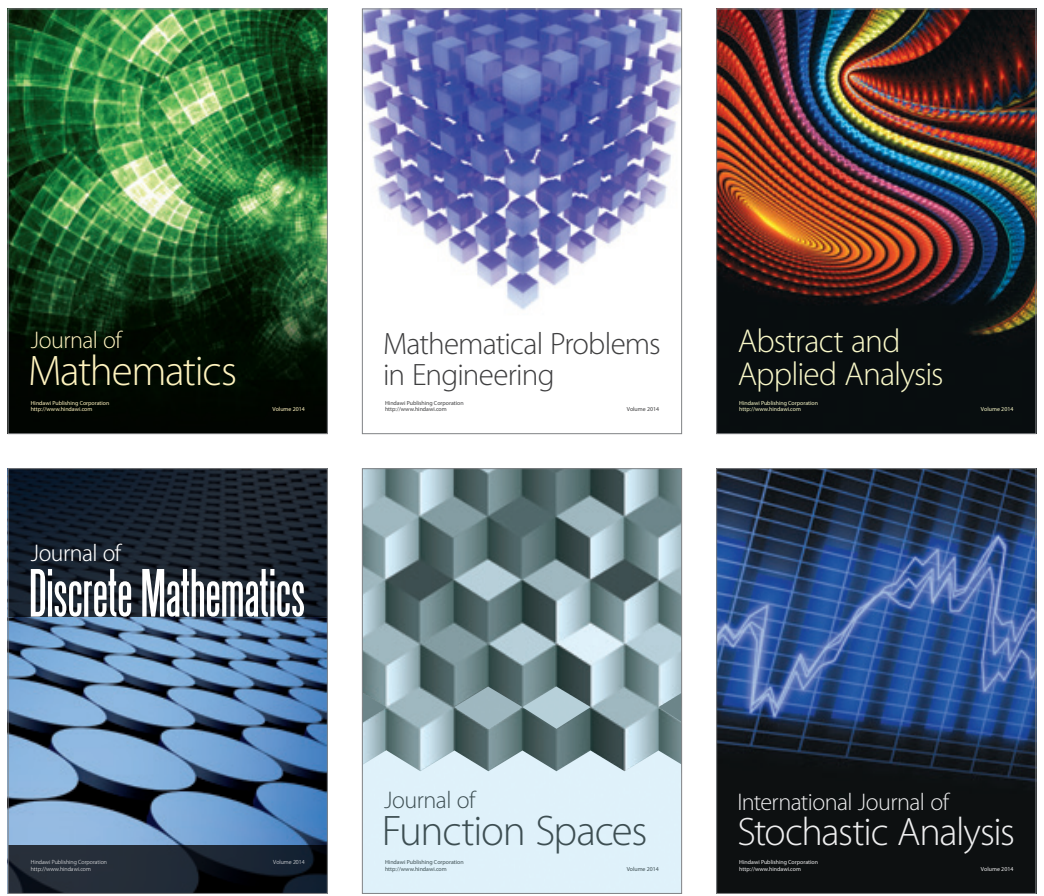

Journal of

Function Spaces

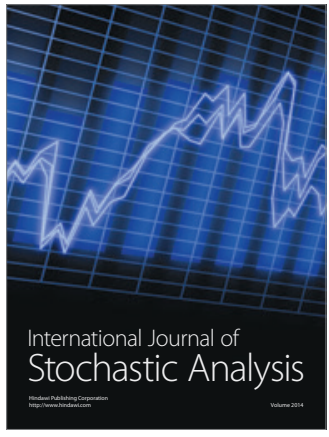

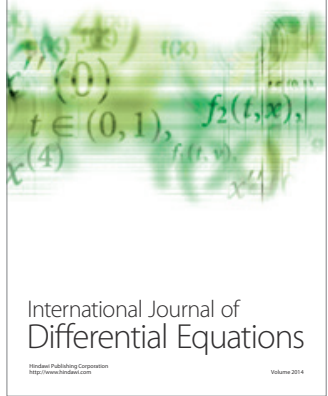
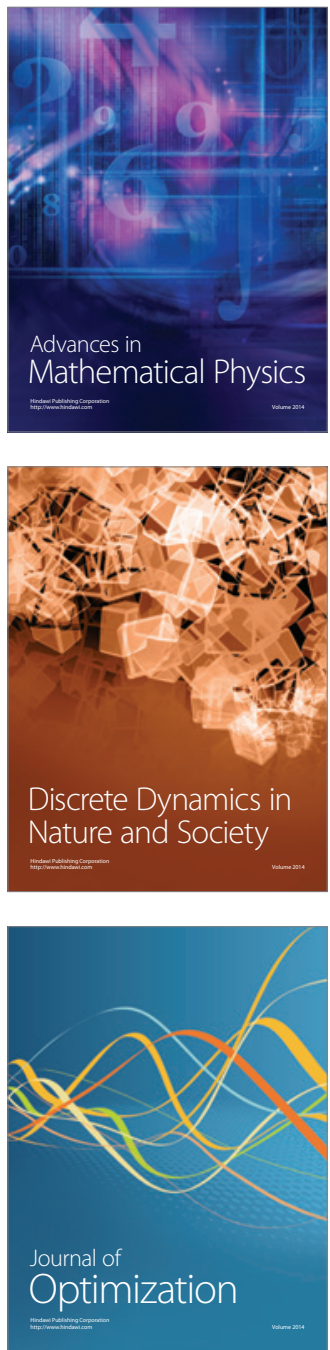